\title{
Quality of life analyses in patients with multiple myeloma: results from the Selinexor (KPT-330) Treatment of Refractory Myeloma (STORM) phase 2b study
}

Gabriel Tremblay ${ }^{1 *}$ (D) Patrick Daniele1, Janis Breeze ${ }^{1}$, Lingling Li², Jatin Shah², Sharon Shacham², Michael Kauffman ${ }^{2}$, Monika Engelhardt $^{3}$, Ajaj Chari $^{4}$, Ajay Nooka ${ }^{5}$, Dan Vogl ${ }^{6}$, Maria Gavriatopoulou ${ }^{7}$, Meletios-Athanasios Dimopoulos ${ }^{8}$, Paul Richardson ${ }^{9}$, Noa Biran ${ }^{10}$, David Siegel ${ }^{10}$, Philip Vlummens ${ }^{11}$, Chantal Doyen ${ }^{12}$, Thierry Facon ${ }^{13}$, Mohamad Mohty ${ }^{14}$, Nathalie Meuleman ${ }^{15}$, Moshe Levy ${ }^{16}$, Luciano Costa ${ }^{17}$, James E. Hoffman ${ }^{18}$, Michel Delforge ${ }^{19}$, David Kaminetzky ${ }^{20}$, Katja Weisel ${ }^{21}$, Marc Raab ${ }^{22}$, David Dingli² ${ }^{23}$, Sascha Tuchman ${ }^{24}$, Frenzel Laurent ${ }^{25}$, Ravi Vij ${ }^{26}$, Gary Schiller ${ }^{27}$, Philippe Moreau ${ }^{28}$, Joshua Richter ${ }^{29}$, Martin Schreder ${ }^{30}$, Klaus Podar ${ }^{31}$, Terri Parker ${ }^{32}$, Robert Frank Cornell ${ }^{33}$, Karlin Lionel ${ }^{34}$, Sylvain Choquet $^{35}$ and Jagannath Sundar ${ }^{29}$

\begin{abstract}
Background: Selinexor is an oral, selective nuclear export inhibitor. STORM was a phase 2b, single-arm, open-label, multicenter trial of selinexor with low dose dexamethasone in patients with penta-exposed relapsed/refractory multiple myeloma (RRMM) that met its primary endpoint, with overall response of 26\% (95\% confidence interval [Cl], 19 to 35\%). Health-related quality of life (HRQoL) was a secondary endpoint measured using the Functional Assessment of Cancer Therapy - Multiple Myeloma (FACT-MM). This study examines impact of selinexor treatment on HRQoL of patients treated in STORM and reports two approaches to calculate minimal clinically important differences for the FACT-MM.

Methods: FACT-MM data were collected at baseline, on day 1 of each 4-week treatment cycle, and at end of treatment (EOT). Changes from baseline were analyzed for the FACT-MM total score, FACT-trial outcome index (TOI), FACT-General (FACT-G), and the MM-specific domain using mixed-effects regression models. Two approaches for evaluating minimal clinically important differences were explored: the first defined as $10 \%$ of the instrument range, and the second based on estimated mean baseline differences between Eastern Cooperative Oncology Group performance status (ECOG PS) scores. Post-hoc difference analysis compared change in scores from baseline to EOT for treatment responders and non-responders.
\end{abstract}

\footnotetext{
* Correspondence: gabrieltremblay@pshta.com

${ }^{1}$ Purple Squirrel Economics, 1600 Notre Dame W, Suite 201, Montreal, QC H3J 1M1, Canada

Full list of author information is available at the end of the article
}

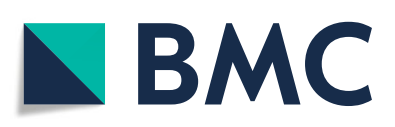

(c) The Author(s). 2021 Open Access This article is licensed under a Creative Commons Attribution 4.0 International License, which permits use, sharing, adaptation, distribution and reproduction in any medium or format, as long as you give appropriate credit to the original author(s) and the source, provide a link to the Creative Commons licence, and indicate if changes were made. The images or other third party material in this article are included in the article's Creative Commons licence, unless indicated otherwise in a credit line to the material. If material is not included in the article's Creative Commons licence and your intended use is not permitted by statutory regulation or exceeds the permitted use, you will need to obtain permission directly from the copyright holder. To view a copy of this licence, visit http://creativecommons.org/licenses/by/4.0/ The Creative Commons Public Domain Dedication waiver (http://creativecommons.org/publicdomain/zero/1.0/) applies to the data made available in this article, unless otherwise stated in a credit line to the data. 
Results: Eighty patients were included in the analysis; the mean number of prior therapies was 7.9 (standard deviation [SD] 3.1), and mean duration of myeloma was 7.6 years (SD 3.4). Each exploratory minimal clinically important difference threshold yielded consistent results whereby most patients did not experience HRQoL decline during the first six cycles of treatment (range: 53.9 to $75.7 \%$ for the first approach; range: 52.6 to $72.9 \%$ for the second). Treatment responders experienced less decline in HRQoL from baseline to EOT than non-responders, which was significant for the FACT-G, but not for other scores.

Conclusion: The majority of patients did not experience decline in HRQOL based on minimal clinically important differences during early cycles of treatment with selinexor and dexamethasone in the STORM trial. An anchor-based approach utilizing patient-level data (ECOG PS score) to define minimal clinically important differences for the FACT-MM gave consistent results with a distribution-based approach.

Trial registration: This trial was registered on ClinicalTrials.gov under the trial-ID NCT02336815 on January 8, 2015.

Keywords: Patient reported outcomes, Health-related quality of life, FACT-MM, Multiple myeloma, Selinexor

\section{Background}

Multiple myeloma (MM) is the second most common form of hematologic cancer in the United States (US), with an estimated 32,110 new cases in 2019 [1]. MM is characterized by the abnormal proliferation of clonal plasma cells in the bone marrow, alterations in the bone marrow microenvironment, and the production of monoclonal protein and other bioactive molecules by malignant cells [2]. Patients with MM experience a burden of symptoms due to clinical manifestations associated with end organ damage, including hypercalcemia, renal insufficiency, renal failure, anemia, immune dysfunction, and bone destruction [3]. Current treatment modalities for MM include proteasome inhibitors, immunomodulatory agents, and monoclonal antibodies, which are often used in doublet or triplet drug regimens, as well as chemotherapy, bone marrow transplant, and radiation therapy $[4,5]$.

According to the US Surveillance, Epidemiology, and End Results (SEER) database, the 5-year survival rate for patients diagnosed with MM from 2010 to 2016 was estimated to be $53.9 \%$ [1]. At present, MM remains generally incurable, and almost all patients relapse and develop refractory disease $[6,7]$. With each relapse, patients face worsening clinical outcomes due to declining efficacy of treatment regimens, shorter duration of response, and increased refractoriness to therapeutic agents $[6,8-10]$.

The refractory nature of MM and severity of symptoms impact quality of life (QoL) and limit availability of treatment options for patients [11-13]. Previous studies have provided evidence of poor QoL among patients with relapsed/refractory MM (RRMM), who face a significant burden of disease and cumulative impacts of prior treatments and treatment-associated adverse events [12]. As patients progress through multiple lines of therapy and exhaust available treatment options with lessening clinical benefit, they may decide between experimental therapy, retreatment strategies, and symptomatic care [14].
Selinexor is a first-in-class selective oral nuclear transport inhibitor that has been approved by the US Food and Drug Administration in combination with low-dose dexamethasone for the treatment of adults with RRMM who have received at least four prior therapies and whose disease is refractory to at least two proteasome inhibitors, two immunomodulatory agents, and an antiCD38 monoclonal antibody [15]. Efficacy and safety of selinexor in RRMM were demonstrated in the STORM (Selinexor Treatment of Refractory Myeloma) phase 2b trial (NCT02336815; $N=122 ; n=83$ with pentarefractory myeloma i.e., refractory to bortezomib, carfilzomib, lenalidomide, pomalidomide, and daratumumab [16]. Results of STORM have been previously published elsewhere [16]. Briefly, the primary endpoint was overall response, defined as a partial response or better, and was observed in $26 \%$ of patients (95\% CI, 19 to $35 \%$ ). Among all responders, the median duration of response was 4.4 months. In the modified intent-to-treat (mITT) population, the median overall survival was 8.6 months. The most common adverse events were thrombocytopenia, nausea, fatigue, anemia, decreased appetite, and decreased weight, which were managed with supportive care and dose modifications. Patient-reported outcome (PRO) data were collected using the Functional Assessment of Cancer Therapy - Multiple Myeloma (FACTMM) instrument at study screening, at each cycle, and at end of treatment. This analysis provides an assessment of patient-reported QoL with selinexor and low dose dexamethasone in the STORM trial. In addition, it aims to evaluate the proportion of patients with minimal clinically meaningful change in QoL from baseline.

\section{Methods}

\section{Study design and quality of life assessment}

The patient eligibility criteria and study design of STORM have been previously described [16]. Briefly, STORM was a phase $2 \mathrm{~b}$, multicenter, open-label study 
of twice-weekly oral selinexor $(80 \mathrm{mg})$ in combination with dexamethasone $(20 \mathrm{mg})$ in patients with progressive MM [16]. The mITT population included 122 patients (median age 65.2 years), of whom 83 (68\%) had pentarefractory MM. Patient-reported QoL was a secondary endpoint and was assessed at study screening and on day 1 of each 4-week treatment cycle beginning at cycle 2 , and at end of treatment with the FACT-MM. The FACT-MM is a disease-specific instrument and has been previously applied in the assessment of health-related QoL (HRQoL) among patients with RRMM in investigational studies [17-20]. FACT-MM combines the General version of the FACT (FACT-G; 27 items) with an MMspecific subscale (MM domain; 14 items). The MM domain addresses symptomatic burden and disease-specific well-being [19]. The total FACT-MM score is obtained by adding individual subscale scores for physical wellbeing (7 items), social/family well-being (7 items), emotional well-being (6 items), and functional well-being domains (7 items) of the FACT-G and the MM domain [19]. The FACT-MM Trial Outcomes Index (TOI) is comprised of the physical and functional subscales and the MM domain [19].

\section{Statistical analysis}

The analysis methods have been previously presented in brief in a conference abstract by Breeze et al. [21]. The QoL analysis dataset consisted of 80 patients in the mITT population with FACT-MM data at baseline and at least one follow-up cycle or end of treatment. Baseline characteristics, including demographic information (e.g., age, gender, race) and clinical variables were summarized using means and standard deviations for continuous variables and counts and proportions for categorical variables. Race was collapsed as white, black, or other for inclusion in the analysis. Patients with missing demographic or clinical variable data were omitted from the HRQoL analyses. Completeness was defined according to the FACT-MM Scoring Guidelines (Version 4), which allows subscales to be calculated if $>50 \%$ of items are present, and total scores if $>80 \%$ of items are present.

For each follow-up cycle, the magnitude of change from baseline was evaluated using mixed-effects regression models, allowing for random slope and intercept terms for repeated measures for the FACT-MM total score, FACT-G, FACT-MM TOI, and the MM domain. This type of regression model assumes data are missing at random (MAR).

Multivariable adjusted models were constructed which considered baseline scores and baseline characteristics including demographic data (age, gender, and race), Eastern Cooperative Oncology Group (ECOG PS) score (categorized as 0 or 1 to 2), and years since diagnosis as prognostic variables. During selection of variables for model inclusion, each model was evaluated for robustness using model fit parameters including Akaike's Information Criterion (AIC), Bayesian Information Criterion (BIC), and the model chi-square statistic [22, 23]. Final adjusted models which include baseline scores and specified baseline characteristics that improved fit are reported.

The minimal clinically important difference represents the smallest meaningful improvement in the score of a PRO domain, interpreted as a minimum level of perceived benefit by patients, and has been generally utilized in the translation of HRQoL outcomes to clinical practice and treatment choice $[24,25]$. To our knowledge, no minimal clinically important difference thresholds have been reported for the FACT-MM. In the current analysis, clinically meaningful changes were evaluated by examination of minimal clinically important difference using two anchor-based approaches. In the first approach, minimal clinically important difference was defined as $10 \%$ of the instrument range, a threshold that has been associated with meaningful HRQoL change in patients using cancer-specific instruments such as the FACT-G [26]. An exploratory approach was developed for this analysis where HRQoL was 'anchored' to differences in ECOG PS scores, which is a measure used by clinicians to assess and describe the clinical status and prognosis of patients and to guide treatment. Previous anchor-based analyses have used clinical characteristics such as ECOG PS or laboratory findings such as hemoglobin levels to derive MCIDs for diseasespecific FACT subscales $[27,28]$. In these analyses, adjacent categories in selected characteristics were presumed to represent clinically distinguishable groups within the HRQoL dataset. Following these approaches, the current analysis used patient-level data to group patients into categories based on physician-assessed baseline ECOG PS. Due to the low number of patients included in the analysis, ECOG PS 1 and 2 categories were grouped together. The minimal clinically important difference was thus defined as the difference in mean baseline scores between patients with ECOG PS of 0 compared with those with ECOG PS of 1 to 2, adjusted for significant baseline characteristics (race, age) to account for confounding arising from the non-randomized nature of the ECOG groupings.

For either approach, patients with a minimal clinically important difference improvement were considered as having HRQoL improvement [24]. Patients with less than the minimal clinically important difference change were considered stable. Patients with a minimal clinically important difference decrease were considered as having HRQoL decline [29].

In addition, post-hoc testing was carried out to examine HRQoL trends between treatment responders and 
non-responders. Responders were defined as patients with overall response (partial response or better). Since these subgroups were not randomized, a difference analysis (a quasi-experimental approach) was used to statistically compare the differences in HRQoL scores from baseline to end of treatment for treatment responders and non-responders. Estimated mean differences between responders and non-responders were directly derived from the mixed effects model.

\section{Results}

The key results of the analysis were previously reported in a conference abstract by Breeze et al. [21]. Of 122 patients in the STORM mITT population, 80 (66\%) completed the FACT-MM at baseline and at one or more follow-up cycle or at end of treatment (Fig. 1). In the QoL analysis population, 21 patients experienced partial response or better and were considered as treatment responders $(26 \% ; n=21 / 80)$. Baseline characteristics and clinical variables for the QoL analysis population are summarized in Table 1 . Patients were heavily pretreated, with a mean number of prior treatments of 7.9 (SD 3.1, range 3 to 18), and a mean duration of myeloma of 7.6 years (SD 3.4, range 1.2 to 18.6). Excluded patients were similar to the HRQoL analysis population with respect to mean age (65.0 vs 63.7 years), mean number of previous regimens ( 7.2 vs 7.9 ), and mean time from initial diagnosis (6.6 vs 7.6 years). Minor differences were noted with respect to sex (62.5 vs $50 \%$ male), proportion of patients with high-risk cytogenetics (59.5 vs $50 \%$ ), and R-ISS risk score stage II (69.0 vs $61.2 \%)$. Given the lack of substantial differences in prognostic factors between the HRQoL analysis population and the excluded patients, the impact of excluding these patients is unlikely to affect the results of the analysis.

Results from the mixed-effects regression analysis for the FACT-MM total score, FACT-G, FACT-MM TOI, and the MM domain are shown in Table 2. Reported coefficients represent the mean change from baseline as estimated by the mixed effects model where a negative value indicates a relative decline from baseline, and a positive value indicates an improvement from baseline. The number of patients who remained in the study declined with each successive cycle, reflecting the highly advanced nature of disease and the proportion of patients who remained well-enough to continue treatment. Most patients showed a monotonic pattern of missingness. Disregarding the end of treatment, only two patients $(2.5 \%)$ showed intermittent missingness. Scores for the FACT-MM, FACT-G and FACT-MM TOI decreased from baseline at each cycle and at the end of treatment, with significant decreases observed in early cycles of treatment as well as end of treatment. The MM domain score did not change significantly at any cycle or at end of treatment.

Next, minimal clinically meaningful changes based on the FACT-MM were evaluated using two anchor-based approaches; the first based on a $10 \%$ difference in scale

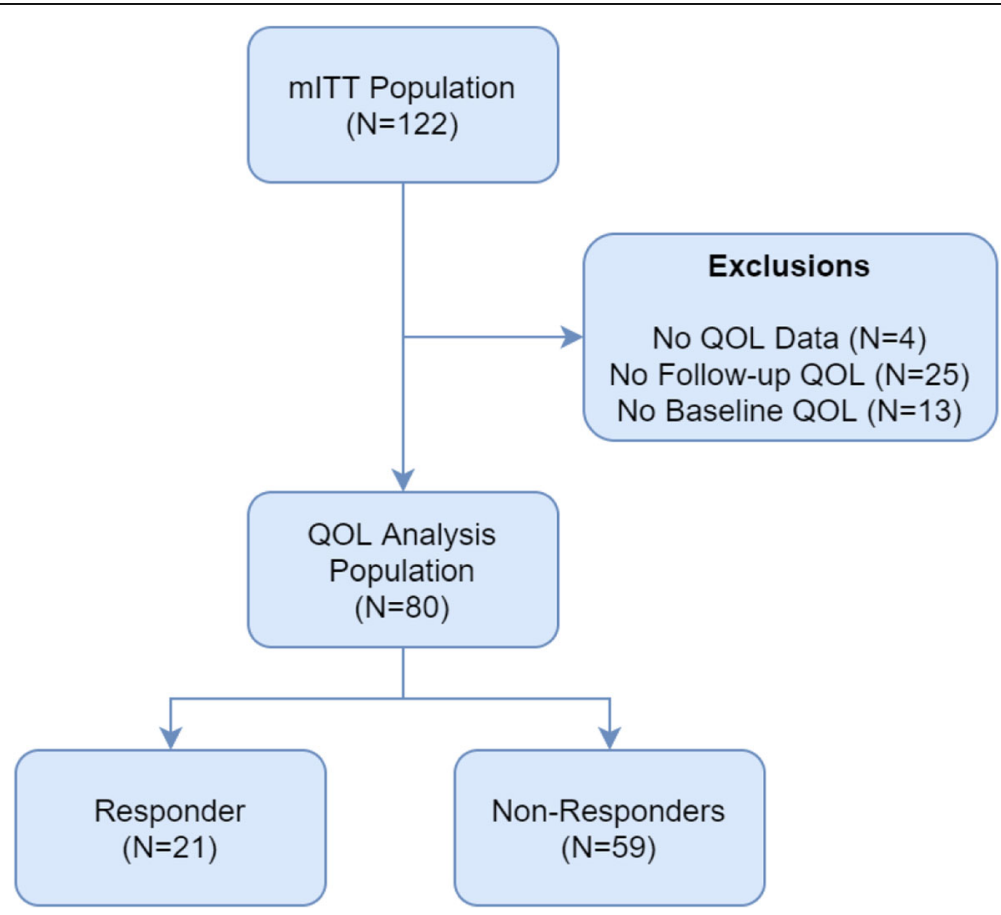

Fig. 1 Study flowchart. mITT: modified intent-to-treat; QoL = quality of life 
Table 1 Patient baseline characteristics

\begin{tabular}{ll}
\hline Patient baseline characteristics & \\
\hline $\mathbf{N}$ (\%) unless otherwise indicated & $\begin{array}{l}\text { QoL analysis } \\
\text { population }(\mathbf{N}=\mathbf{8 0})\end{array}$ \\
\hline Male & $50(62.5)$ \\
Mean age (SD), range & $63.7(9.4), 40.4$ to 85.9 \\
Race & \\
White & $57(71)$ \\
Black & $8(10)$ \\
Other & $11(14)$ \\
Missing & $4(5)$ \\
ECOG performance status & \\
0 & $25(31)$ \\
1 & $45(56)$ \\
2 & $7(9)$ \\
Missing & $3(4)$ \\
R-ISS & \\
1 & $16(20)$ \\
2 & $49(61)$ \\
3 & $14(18)$ \\
Missing & $1(1)$ \\
High-risk cytogenetic abnormalities (any of & $40(50)$ \\
Mean number of previous regimens (SD), range & $7.9(3.1), 3$ to 18 \\
Mean years since diagnosis (SD), range & $7.6(3.4), 1.2$ to 18.6 \\
\hline
\end{tabular}

QoL quality of life, ECOG Eastern Cooperative Oncology Group, R-ISS Revised International Staging System, SD standard deviation range, and the second based on mean baseline differences between ECOG groups, adjusted for baseline characteristics of age and race. The minimal clinically important difference thresholds calculated for the two approaches are shown in Table 3. These thresholds can be interpreted as the smallest clinically meaningful change for a particular domain. For example, a decrease of the FACT-MM total score by 13.5 points from baseline would represent a clinically meaningful decline as defined by the ECOG-based anchor.

The number and proportion of patients who improved, experienced no change, or declined in HRQoL based on the minimal clinically important difference anchored by ECOG groups is shown in Table 4. The results of the analysis based on minimal clinically important difference anchored by a $10 \%$ difference in scale range is shown in the Supplementary Information. The combined proportions of patients who experienced no change in HRQoL or improvements compared to baseline through cycle 6 based on the FACT-MM total score, FACT-G, FACT-MM TOI, and the MM domain were generally greater than the proportions who experienced declines. Results of the analyses, according to the two minimal clinically important difference definitions, were consistent.

Post-hoc analysis evaluated trends in HRQoL change from baseline to end of treatment between treatment responders and non-responders using a mixed-effects model. It should be noted that in the QoL dataset, there were 21 responders $(26 \% ; n=21 / 80)$, suggesting that responders were no more likely than non-responders to

Table 2 Change in HRQoL as evaluated by a mixed-effects regression model

\begin{tabular}{|c|c|c|c|c|c|c|c|c|c|}
\hline & \multirow[b]{2}{*}{$\begin{array}{l}\text { Max } \\
\mathbf{N}\end{array}$} & \multicolumn{2}{|c|}{$\begin{array}{l}\text { Change in FACT-MM total } \\
\text { score }^{\mathrm{a}}\end{array}$} & \multicolumn{2}{|c|}{ Change in FACT-G score ${ }^{a}$} & \multicolumn{2}{|c|}{$\begin{array}{l}\text { Change in FACT-MM TOI } \\
\text { total score }\end{array}$} & \multicolumn{2}{|c|}{$\begin{array}{l}\text { Change in MM domain } \\
\text { score }^{c}\end{array}$} \\
\hline & & Coefficient $(95 \% \mathrm{Cl})$ & $\begin{array}{l}p- \\
\text { value }\end{array}$ & $\begin{array}{l}\text { Coefficient }(95 \% \\
\text { Cl) }\end{array}$ & $\begin{array}{l}p \text { - } \\
\text { value }\end{array}$ & $\begin{array}{l}\text { Coefficient }(95 \% \\
\mathrm{CI})\end{array}$ & $\begin{array}{l}p- \\
\text { value }\end{array}$ & $\begin{array}{l}\text { Coefficient }(95 \% \\
\text { CI) }\end{array}$ & $\begin{array}{l}p- \\
\text { value }\end{array}$ \\
\hline Cycle 2 & $71^{d}$ & $-3.94(-8.03$ to 0.16$)$ & 0.059 & $\begin{array}{l}-3.87(-6.66 \text { to } \\
-1.08)\end{array}$ & 0.007 & $\begin{array}{l}-4.86(-8.44 \text { to } \\
-1.29)\end{array}$ & 0.008 & $\begin{array}{l}-0.10(-2.11 \text { to } \\
1.92)\end{array}$ & 0.926 \\
\hline Cycle 3 & 42 & $\begin{array}{l}-5.17(-10.10 \text { to } \\
-0.25)\end{array}$ & 0.040 & $\begin{array}{l}-5.69(-9.05 \text { to } \\
-2.33)\end{array}$ & 0.001 & $\begin{array}{l}-4.92(-9.21 \text { to } \\
-0.64)\end{array}$ & 0.024 & $0.61(-1.81$ to 3.03$)$ & 0.619 \\
\hline Cycle 4 & 25 & $-5.47(-11.38$ to 0.45$)$ & 0.070 & $-4.00(-8.04$ to 0.04$)$ & 0.052 & $\begin{array}{l}-5.65(-10.79 \text { to } \\
-0.52)\end{array}$ & 0.031 & $\begin{array}{l}-1.35(-4.28 \text { to } \\
1.58)\end{array}$ & 0.367 \\
\hline Cycle 5 & 13 & $-3.97(-11.58$ to 3.65$)$ & 0.307 & $-3.62(-8.82$ to 1.59$)$ & 0.173 & $\begin{array}{l}-4.60(-11.22 \text { to } \\
2.03)\end{array}$ & 0.174 & $0.21(-3.43$ to 3.85$)$ & 0.911 \\
\hline Cycle 6 & $8^{e}$ & $-3.54(-13.59$ to 6.51$)$ & 0.490 & $\begin{array}{l}-4.62(-11.49 \text { to } \\
2.25)\end{array}$ & 0.187 & $\begin{array}{l}-3.35(-12.10 \text { to } \\
5.41)\end{array}$ & 0.453 & $2.57(-1.95$ to 7.09$)$ & 0.265 \\
\hline $\begin{array}{l}\text { End of } \\
\text { treatment }\end{array}$ & $39^{f}$ & $\begin{array}{l}-10.45(-16.19 \text { to } \\
-4.70)\end{array}$ & $\begin{array}{l}< \\
0.0001\end{array}$ & $\begin{array}{l}-8.14(-11.68 \text { to } \\
-4.60)\end{array}$ & $\begin{array}{l}< \\
0.001\end{array}$ & $\begin{array}{l}-9.39(-14.22 \text { to } \\
-4.57)\end{array}$ & $\begin{array}{l}< \\
0.001\end{array}$ & $\begin{array}{l}-1.80(-4.93 \text { to } \\
1.34)\end{array}$ & 0.261 \\
\hline
\end{tabular}

AIC Akaike's Information Criterion, BIC Bayesian Information Criterion, FACT-G Functional Assessment of Cancer Therapy - General, FACT-MM Functional Assessment of Cancer Therapy - Multiple Myeloma, MM multiple myeloma, TOI Trial Outcomes Index, $\mathrm{Cl}$ confidence interval

${ }^{a}$ Adjusted for baseline score, race, sex, and years since diagnosis

${ }^{b}$ Adjusted for baseline score, race, sex, and number of prior regimens

c Adjusted for baseline score, race, sex, ECOG performance score, and number of prior regimens

${ }^{d} n=70$ for FACT-MM total score and FACT-G

e $n=7$ for FACT-MM total score, FACT-G, and FACT-TOI

${ }^{\mathrm{f}} n=38$ for FACT-MM total score, FACT-G, and FACT-TOI 
Table 3 Minimal clinically important difference thresholds for two anchor-based approaches

\begin{tabular}{lll}
\hline & $\mathbf{1 0} \%$ difference in score range & Mean baseline difference between ECOG performance scores $\mathbf{0}$ vs $\mathbf{1}$ to $\mathbf{2}$ \\
\hline Total FACT-MM & 16.4 & 13.5 \\
FACT-G & 10.8 & 7.8 \\
FACT-TOI & 8.4 & 11.0 \\
MM Domain & 5.6 & 5.8 \\
\hline
\end{tabular}

ECOG Eastern Cooperative Oncology Group, FACT-G Functional Assessment of Cancer Therapy - General, FACT-MM Functional Assessment of Cancer Therapy Multiple Myeloma, MM multiple myeloma, TOI Trial Outcomes Index

complete the FACT-MM assessment [16]. Results of the difference analysis are summarized in Table 5 . With the exception of the MM domain, the negative values for mean change indicate that HRQoL was decreasing for responders and non-responders. The FACT-MM, FACT-G, FACT-MM TOI, and the MM domain scores of non-responders showed a greater decrease from baseline to end of treatment. In contrast, responders had no change as evidenced by positive values of the mean difference. This observed mean difference was significant for the FACT-G $(p=0.043)$, but not other scales.

\section{Discussion}

Despite advances made in the treatment of RRMM, the disease remains incurable and patients with RRMM face a significant burden due to symptoms, treatmentassociated adverse events, and cumulative toxicities of prior therapies [11-13]. Inclusion of QoL evidence is an important factor in treatment decision-making that aims to balance clinical efficacy of newer therapies with the burden of adverse events, particularly among heavily pretreated patients with advanced disease [13]. Several studies have examined HRQoL in patients with RRMM receiving doublet or triplet therapies, daratumumab, or autologous stem cell transplantation with a variety of disease specific instruments [17, 30-39]. Maintenance in HRQoL was observed in randomized controlled trials (RCTs) with pomalidomide and low-dose dexamethasone [39], panobinostat, bortezomib, and dexamethasone [36], daratumumab [31], pomalidomide, bortezomib, and dexamethasone [38], and carfilzomib and dexamethasone [34] in later lines of therapy $(3 \mathrm{~L}+)$.

The current analysis examined HRQoL effects in patients with penta-refractory MM, who received selinexor and low dose dexamethasone in the STORM phase $2 \mathrm{~b}$ trial. FACT-MM, FACT-G, and FACT-MM TOI scores of patients declined significantly from baseline in the early cycles and at the end of treatment, while significant changes were not observed in the MM-specific domain at any point from baseline. Two anchors were utilized to estimate minimal clinically important difference and yielded consistent findings. Anchor-based approaches have been utilized to establish minimal clinically important difference for other FACT instruments [40-44]. No known, validated minimal clinically important difference has been reported for the FACT-MM. The exploratory approach utilizing patient-level data was based on the previously established relationship between QoL outcomes and ECOG PS scores [20]. This is in contrast to distribution-based minimal clinically important difference evaluations, which do not consider clinical reference points and are only statistical by nature. The observed consistency in findings with the previously applied distribution-based approach serves as a validation of the novel anchor-based approach.

A key finding of the analysis is that, generally, the combined proportions of patients who experienced no change in HRQoL or improvements were higher compared to those who experienced declines in the early cycles of treatment with selinexor and dexamethasone according to minimal clinically important differences. In addition, the difference analysis identified that treatment responders had less HRQoL decline than nonresponders. An association between HRQoL and response to treatment has been observed in previous RCTs. A significant improvement in HRQoL was observed in patients with partial or complete response with bortezomib in the SUMMIT phase 2 trial, while deterioration in HRQoL was observed among patients who did not respond and had progressive disease based on the European Organisation for Research and Treatment of Cancer Quality of Life Questionnaire C-30 (EORTC QLQ-C30) [45]. In the carfilzomib, lenalidomide, and dexamethasone arm of the phase 3 RCT ASPIRE, patients achieving a partial response or better had significantly higher HRQoL over 18 cycles of treatment compared with patients who did not respond to treatment, according to the Global Health Status scale of the EORTC QLQ-C30 [37]. The limited impact on HRQoL, particularly among treatment responders, suggests a favorable benefit-risk profile of selinexor, given its demonstrated efficacy and tolerability among patients with penta-refractory MM, and considering the unmet therapeutic need in this patient population.

\section{Limitations}

An important limitation of the analysis is the single-arm study design of the STORM phase $2 \mathrm{~b}$ trial, which did 


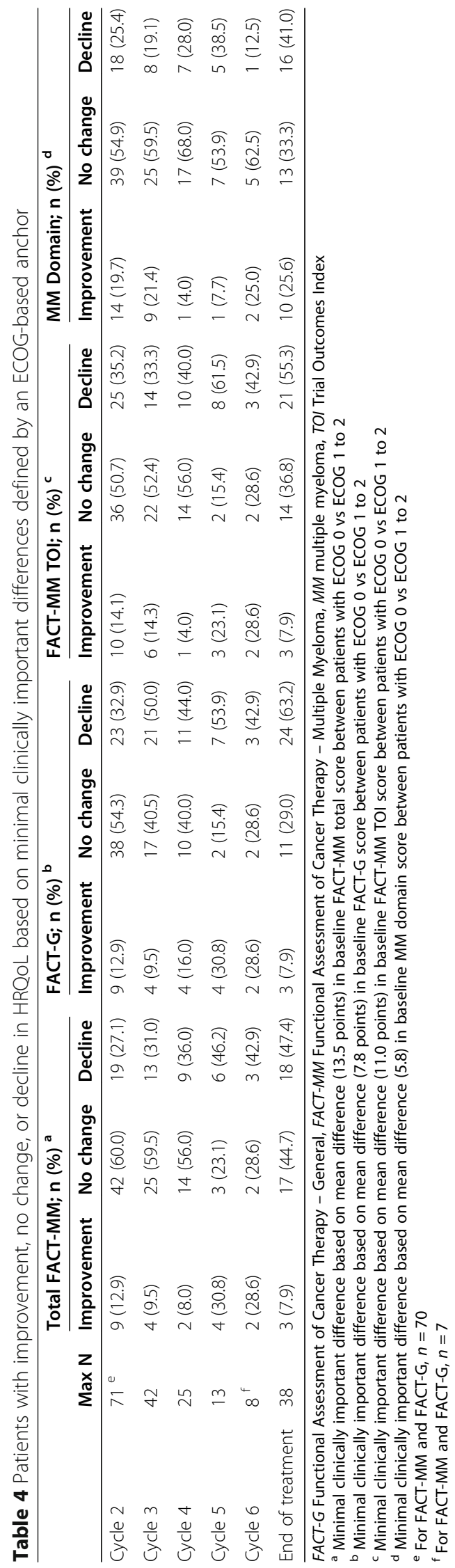


Table 5 Difference analysis results

\begin{tabular}{|c|c|c|c|}
\hline & $\begin{array}{l}\text { Responders } \\
(N=21)\end{array}$ & $\begin{array}{l}\text { Non-responders } \\
(N=59)\end{array}$ & Mean difference $^{a}$ \\
\hline & Mean change from baseline $(95 \% \mathrm{Cl})$ & Mean change from baseline $(95 \% \mathrm{Cl})$ & $\begin{array}{l}(95 \% \mathrm{Cl}) \\
p \text {-value }\end{array}$ \\
\hline Total FACT-MM & $-1.72(-13.63$ to 10.20$)$ & $-12.89(-19.18$ to -6.60$)$ & $\begin{array}{l}11.17(-2.30 \text { to } 24.65) \\
p=0.104\end{array}$ \\
\hline FACT-G & $-3.05(-8.90$ to 2.79$)$ & $-10.28(-14.13$ to -6.42$)$ & $\begin{array}{l}7.22(0.22 \text { to } 14.22) \\
p=0.043\end{array}$ \\
\hline FACT-MM TOI & $-4.33(-14.11$ to 5.44$)$ & $-10.60(-15.84$ to -5.35$)$ & $\begin{array}{l}6.26(-4.82 \text { to } 17.35) \\
p=0.268\end{array}$ \\
\hline MM Domain & $2.57(-3.05$ to 8.19$)$ & $-2.16(-5.60$ to 1.27$)$ & $\begin{array}{l}4.73(-1.85 \text { to } 11.32) \\
p=0.159\end{array}$ \\
\hline
\end{tabular}

FACT-G Functional Assessment of Cancer Therapy - General, FACT-MM Functional Assessment of Cancer Therapy - Multiple Myeloma, MM multiple myeloma, TOI Trial Outcomes Index, $\mathrm{Cl}$ confidence interval

${ }^{a}$ Difference analysis between baseline and end of treatment in responders compared to non-responders as estimated by a mixed effects model

not enable comparison of HRQoL outcomes with a comparator arm who received conventional medical management. As a result, treatment-associated changes in HRQoL cannot be directly extracted from the analysis. HRQoL was a secondary endpoint, and all analyses and results should be considered as explorative. The analysis aimed to examine change in HRQoL during, and at end of treatment with selinexor and low dose dexamethasone. The analysis did not investigate treatmentassociated adverse events that may have been associated with changes in HRQoL due to the small sample size of the HRQoL dataset and high attrition rates seen in STORM; considering these factors, the study would be underpowered to detect significant differences.

Another limitation of the analysis was the small sample size of patients with post-baseline HRQoL data. The sample size decreased over time, particularly in later cycles (i.e., cycle 7 or greater) that were omitted from the analysis due to sparse numbers. Observed differences in the composition of patients with minimal clinically important differences may be attributed to attrition, particularly among non-responders. Compliance rates were good in earlier cycles up to cycle 5 , with $\geq 65 \%$ of patients on treatment completing the FACT-MM, however a decline to $54 \%$ was observed in cycle 6 . Combined with the small sample size, statistical power to detect changes in scores may be further reduced for each covariate added to the model.

The mixed-effects models assumed an MAR pattern of data, which presumes that all characteristics associated with missingness were adjusted for in the model. The MAR assumption was tested by examining patterns of missingness in the trial. Because different groups of patients were observed at each cycle, baseline FACT-MM scores also varied across each cycle. The moving baseline values and the MAR assumption should be taken into consideration in the interpretation of observed HRQoL changes.
Lastly, treatment responders were not randomized. As a result, significant differences between responders and non-responders could be present between HRQoL at baseline or for other baseline characteristics. A difference analysis was performed since subgroup analysis according to response was not well powered to perform statistical testing. It should be noted that the difference analysis is a quasi-experimental approach, which has been utilized in epidemiologic studies, but has not been commonly used for HRQoL analyses [46].

\section{Conclusions}

The current analysis examined patient-reported HRQoL in the STORM mITT population using the FACT-MM. Minimal clinically important difference analyses demonstrated that most patients did not experience HRQoL decline during early cycles of treatment with selinexor and low dose dexamethasone. Exploratory minimal clinically important differences, defined as $10 \%$ of the instrument range or as an ECOG-based anchor, yielded consistent results. Treatment responders were found to experience less decline in HRQoL from baseline to end of treatment than non-responders, which was significant only for the FACT-G. Important limitations of the analysis were the single-arm study design and the limited sample size. Overall findings complement the demonstrated efficacy and tolerability of selinexor with low dose dexamethasone in patients with penta-refractory MM.

\footnotetext{
Abbreviations

AIC: Akaike's Information Criterion; BIC: Bayesian Information Criterion; Cl: Confidence interval; ECOG: Eastern Cooperative Oncology Group; EORTC QLQ-C30: European Organisation for Research and Treatment of Cancer Quality of Life Questionnaire C-30; FACT-G: Functional Assessment of Cancer Therapy - General; FACT-MM: Functional Assessment of Cancer Therapy Multiple Myeloma; FACT-TOI: Functional Assessment of Cancer Therapy Trial Outcomes Index; HRQoL: Health-related quality of life; MAR: Missing at random; mITT: Modified intent-to-treat; MM: Multiple myeloma; QoL: Quality of life; RCT: Randomized controlled trial; R-ISS: Revised International Staging
} 
System; RRMM: Relapsed/refractory multiple myeloma; SD: Standard deviation; STORM: Selinexor Treatment of Refractory Myeloma

\section{Supplementary Information}

The online version contains supplementary material available at https://doi. org/10.1186/s12885-021-08453-9.

Additional file $\mathbf{1}$ Table 1. Patients with improvement, no change, or decline in $\mathrm{HRQOL}$ based on minimal clinically important differences defined by $\geq 10 \%$ of the instrument range. Table $\mathbf{1}$ describes the number and proportion of patients with improvement, no change, or decline in HRQoL based on the minimal clinically important difference threshold defined as $\geq 10 \%$ of the instrument range. Data are shown for the FACT-MM, FACT-G, FACT-MM TOI, and the MM domain at treatment cycle 2-6 and end of treatment.

Additional file 2. Provides a list of IECS and IRBS for the STORM trial.

\section{Acknowledgements}

KARE council members provided helpful comments on the manuscript. The authors acknowledge and thank the patients who participated in the NCT02336815 trial, their families, caregivers, and the study staff and healthcare providers at all trial sites. Writing and editorial support was provided by Nesrin Vurgun and Karen Sandman, who are employees of Purple Squirrel Economics.

\section{Authors' contributions}

G.T., J.B. and P.D. were responsible for data analyses and data interpretation and participated in drafting and revising of the manuscript. L.L., J.S., S.S., M.K., M.E., A.C., A.N., D.V., M.G., MA.D., P.R., N.B., D.S., P.V., C.D., T.F., M.M., N.M., M.L., L.C., JE.H., M.D., D.K., K.W., M.R., D.D., S.T., F.L., R.V., G.S., P. M, J.R., M.S., K.P., T.P., RF.C., K.L., S.C. and J.S. were responsible for study conception and design and participated in data interpretation and reviewed the manuscript. All authors have read and approved the manuscript.

\section{Funding}

This study and work were funded by Karyopharm Therapeutics Inc.

\section{Availability of data and materials}

The datasets analyzed in this work may be available from the corresponding author on reasonable request and permission of Karyopharm Therapeutics Inc.

\section{Declarations}

\section{Ethics approval and consent to participate}

This study was performed in compliance with the ethical principles that originate from the Declaration of Helsinki and are consistent with the International Council for Harmonisation (ICH) guidelines on Good Clinical Practice (GCP) and regulatory requirements as applicable. The study protocol was approved by the institutional review board or an independent ethics committee at each study center. Written informed consent in accordance with federal, local, and institutional guidelines was obtained from all patients. A list of Institutional Ethics Committees (IECs) and Institutional Review Boards (IRBs) is provided as a supplement.

\section{Consent for publication}

Not applicable.

\section{Competing interests}

Gabriel Tremblay, Janis Breeze and Patrick Daniele are employees of Purple Squirrel Economics, which received funding from Karyopharm Therapeutics Inc. to conduct the analysis. Lingling Li, Jatin Shah, Sharon Shacham, Michael Kauffman are employees of Karyopharm Therapeutics Inc. All other authors were investigators in the STORM trial.

\section{Author details}

'Purple Squirrel Economics, 1600 Notre Dame W, Suite 201, Montreal, QC H3J 1M1, Canada. ${ }^{2}$ Karyopharm Therapeutics Inc., Newton, USA. ${ }^{3}$ University of Freiburg, Freiburg im Breisgau, Germany. ${ }^{4}$ Icahn School of Medicine at
Mount Sinai, New York, USA. ${ }^{5}$ Winship Cancer Institute, Emory University, Atlanta, USA. ${ }^{6}$ Abramson Cancer Center, Perelman School of Medicine, University of Pennsylvania, Philadelphia, USA. ' Oncology Department, Alexandra Hospital, Athens, Greece. ${ }^{8}$ School of Medicine, National and Kapodistrian University of Athens, Athens, Greece. ${ }^{9}$ Harvard Cancer Center, Boston, USA. ${ }^{10}$ Hackensack Meridian Health Hackensack University Medical Center, Hackensack, USA. " University Hospital Ghent, Ghent, Belgium.

${ }^{12}$ Université catholique de Louvain, Ottignies-Louvain-la-Neuve, Belgium.

${ }^{13}$ University Hospital, Lille, France. ${ }^{14}$ Hopital Saint-Antoine, Paris, France.

${ }^{15}$ Institut Jules Bordet, Brussels, Belgium. ${ }^{16}$ Baylor University Medical Center, Dallas, USA. ${ }^{17}$ University of Alabama at Birmingham, Birmingham, USA.

${ }^{18}$ Sylvester Cancer Center, University of Miami, Miami, USA. ${ }^{19}$ University of Leuven, Leuven, Belgium. ${ }^{20}$ New York University Langone Medical Center, New York, USA. ${ }^{21}$ University Medical Center Hamburg-Eppendorf, Hamburg, Germany. ${ }^{22}$ University of Heidelberg, Heidelberg, Germany. ${ }^{23}$ Mayo Clinic, Rochester, USA. ${ }^{24}$ Lineberger Comprehensive Cancer Center at University of North Carolina-Chapel Hill, Chapel Hill, USA. ${ }^{25}$ Hôpital Necker, Paris, France.

${ }^{26}$ Washington University School of Medicine, St. Louis, USA. ${ }^{27}$ David Geffen School of Medicine at University of California, Los Angeles, USA. ${ }^{28}$ University of Nantes, Nantes, France. ${ }^{29}$ Tisch Cancer Institute, Icahn School of Medicine at Mount Sinai, New York, USA. ${ }^{30}$ University Hospital Würzburg, Würzburg, Germany. ${ }^{31}$ University Hospital Krems, Karl Landsteiner University of Health Sciences, Krems an der Donau, Austria. ${ }^{32}$ Yale School of Medicine, New Haven, USA. ${ }^{33}$ Vanderbilt University Medical Center, Nashville, USA. ${ }^{34}$ Centre Hospitalier Lyon Sud, Saint-Genis-Laval, France. ${ }^{35}$ La Pitié Salpêtrière Hospital, Paris, France.

Received: 13 November 2020 Accepted: 7 June 2021

Published online: 06 September 2021

\section{References}

1. Surveillance, Epidemiology, and End Results (SEER) Program. Cancer stat facts: myeloma: National Cancer Institute (NCI); 2016. [cited 2018 Nov 27]. Available from: https://seer.cancer.gov/statfacts/html/mulmy.html

2. Rajkumar SV, Kumar S. Multiple myeloma: diagnosis and treatment. Mayo Clin Proc. 2016;91(1):101-19. https://doi.org/10.1016/j.mayocp.2015.11.007.

3. Bazarbachi AH, Al Hamed R, Malard F, Harousseau J-L, Mohty M. Relapsed refractory multiple myeloma: a comprehensive overview. Leukemia. 2019; 33(10):2343-57. https://doi.org/10.1038/s41375-019-0561-2.

4. Sonneveld P, De Wit E, Moreau P. How have evolutions in strategies for the treatment of relapsed/refractory multiple myeloma translated into improved outcomes for patients? Crit Rev Oncol/Hematol. 2017;112:153-70.

5. Mikhael J. Treatment options for triple-class refractory multiple myeloma. Clin Lymphoma Myeloma Leuk. 2020;20(1):1-7. https://doi.org/10.1016/j. clml.2019.09.621.

6. Kumar SK, Dimopoulos MA, Kastritis E, Terpos E, Nahi H, Goldschmidt H, et al. Natural history of relapsed myeloma, refractory to immunomodulatory drugs and proteasome inhibitors: a multicenter IMWG study. Leukemia. 2017;31(11):2443-8. https://doi.org/10.1038/leu.2017.138.

7. Kumar S, Paiva B, Anderson KC, Durie B, Landgren O, Moreau P, et al. International Myeloma Working Group consensus criteria for response and minimal residual disease assessment in multiple myeloma. Lancet Oncol. 2016;17(8):e328-46. https://doi.org/10.1016/S1470-2045(16)30206-6.

8. Cornell RF, Gandhi UH, Lakshman A, Gahvari Z, Jagosky MH, McGehee E, et al. Subsequent treatment outcomes of multiple myeloma refractory to CD38-monoclonal antibody therapy. Blood. 2018;132(Supplement 1):2015.

9. Gandhi UH, Cornell RF, Lakshman A, Gahvari ZJ, McGehee E, Jagosky MH, et al. Outcomes of patients with multiple myeloma refractory to CD38targeted monoclonal antibody therapy. Leukemia. 2019;33(9):2266-75. https://doi.org/10.1038/s41375-019-0435-7.

10. Pick M, Vainstein V, Goldschmidt N, Lavie D, Libster D, Gural A, et al. Daratumumab resistance is frequent in advanced-stage multiple myeloma patients irrespective of CD38 expression and is related to dismal prognosis. Eur J Haematol. 2018;100(5):494-501. https://doi.org/10.1111/ejh.13046.

11. Hulin C, Hansen T, Heron L, Pughe R, Streetly M, Plate A, et al. Living with the burden of relapse in multiple myeloma from the patient and physician perspective. Leuk Res. 2017;59:75-84. https://doi.org/10.1016/j.leukres.2017.05.019.

12. Ramsenthaler C, Osborne TR, Gao W, Siegert RJ, Edmonds PM, Schey SA, et al. The impact of disease-related symptoms and palliative care concerns on health-related quality of life in multiple myeloma: a multi-centre study. BMC Cancer. 2016;16(1):427. https://doi.org/10.1186/s12885-016-2410-2. 
13. Sonneveld P, Verelst SG, Lewis P, Gray-Schopfer V, Hutchings A, Nixon A, et al. Review of health-related quality of life data in multiple myeloma patients treated with novel agents. Leukemia. 2013;27(10):1959-69. https:// doi.org/10.1038/leu.2013.185.

14. NCCN Clinical Practice Guidelines in Oncology (NCCN Guidelines). Multiple myeloma: National Comprehensive Cancer Network; 2019. [cited 2020 Feb 14]. Available from: https:/www.nccn.org/professionals/physician_gls/pdf/myeloma.pdf

15. XPOVIO (selinexor) prescribing information. [cited $2019 \mathrm{Jul} 8$ ]. Available from: https://www.accessdata.fda.gov/drugsatfda_docs/label/2019/212306s000lbl.pdf.

16. Chari A, Vogl DT, Gavriatopoulou M, Nooka AK, Yee AJ, Huff CA, et al. Oral selinexor-dexamethasone for triple-class refractory multiple myeloma. N Engl J Med. 2019;381(8):727-38. https://doi.org/10.1056/NEJMoa1903455

17. Abonour R, Wagner L, Durie BGM, Jagannath S, Narang M, Terebelo HR, et al. Impact of post-transplantation maintenance therapy on health-related quality of life in patients with multiple myeloma: data from the connect $^{\circledR}$ MM registry. Ann Hematol. 2018;97(12):2425-36. https://doi.org/10.1007/ s00277-018-3446-y.

18. Wagner LI, Robinson D, Weiss M, Katz M, Greipp P, Fonseca R, et al. Content development for the functional assessment of cancer therapy-multiple myeloma (FACT-MM): use of qualitative and quantitative methods for scale construction. J Pain Symptom Manag. 2012;43(6):1094-104. https://doi.org/1 0.1016/j.jpainsymman.2011.06.019

19. Webster K, Cella D, Yost K. The functional assessment of chronic illness therapy (FACIT) measurement system: properties, applications, and interpretation. Health Qual Life Outcomes. 2003;1(1):79. https://doi.org/10.11 86/1477-7525-1-79.

20. Weiss M, Jacobus S, Wagner LI, Cella D, Katz MS, Rajkumar V, et al. Development of the functional assessment of cancer therapy-multiple myeloma (FACT-MM) scale and validation in the eastern cooperative oncology group trial E1A05. Blood. 2011;118(21):4184. https://doi.org/10.11 82/blood.V118.21.4184.4184.

21. Breeze J, Mehta J, Tremblay G, Shacham S, Shah J, Li L, et al. PCN304 quality of LIFE analyses in patients with multiple myeloma: results from the selinexor (KPT-330) treatment of refractory myeloma (STORM) phase 2B study. Value Health. 2020;23:S77. https://doi.org/10.1016/j.jval.2020.04.1767.

22. Akaike $\mathrm{H}$. A new look at the statistical model identification. IEEE Trans Automat Contr. 1974;19(6):716-23. https://doi.org/10.1109/TAC.1974.1100705.

23. Schwarz G. Estimating the dimension of a model. Ann Stat. 1978;6(2):461-4.

24. Jaeschke R, Singer J, Guyatt GH. Measurement of health status. Control Clin Trials. 1989;10(4):407-15. https://doi.org/10.1016/0197-2456(89)90005-6.

25. Jayadevappa R, Cook R, Chhatre S. Minimal important difference to infer changes in health-related quality of life-a systematic review. J Clin Epidemiol. 2017;89:188-98. https://doi.org/10.1016/j.jclinepi.2017.06.009.

26. Ringash J, O'Sullivan B, Bezjak A, Redelmeier DA. Interpreting clinically significant changes in patient-reported outcomes. Cancer. 2007;110(1):196202. https://doi.org/10.1002/cncr.22799.

27. Cella D, Eton DT, Lai J-S, Peterman AH, Merkel DE. Combining anchor and distribution-based methods to derive minimal clinically important differences on the functional assessment of cancer therapy (FACT) Anemia and fatigue scales. J Pain Symptom Manag. 2002;24(6):547-61. https://doi. org/10.1016/S0885-3924(02)00529-8.

28. Pickard AS, Neary MP, Cella D. Estimation of minimally important differences in EQ-5D utility and VAS scores in cancer. Health Qual Life Outcomes. 2007 Dec;5(1):70. https://doi.org/10.1186/1477-7525-5-70.

29. Janelsins MC, Heckler CE, Peppone LJ, Kamen C, Mustian KM, Mohile SG, et al. Cognitive complaints in survivors of breast cancer after chemotherapy compared with age-matched controls: an analysis from a nationwide, multicenter, prospective longitudinal study. J Clin Oncol. 2017;35(5):506-14. https://doi.org/10.1200/JCO.2016.68.5826.

30. Ahmedzai SH, Snowden JA, Ashcroft AJ, Cairns DA, Williams C, Hockaday A, et al. Patient-reported outcome results from the open-label, randomized phase III myeloma $X$ trial evaluating salvage autologous stem-cell transplantation in relapsed multiple myeloma. JCO. 2019;37(19):1617-28. https://doi.org/10.1200/JCO.18.01006.

31. Chari A, Lonial S, Mark TM, Krishnan AY, Stockerl-Goldstein KE, Usmani SZ, et al. Results of an early access treatment protocol of daratumumab in United States patients with relapsed or refractory multiple myeloma: early access treatment with daratumumab. Cancer. 2018;124(22):4342-9. https:// doi.org/10.1002/cncr.31706.

32. Kim SJ, Park Y, Mun Y-C, Kim JS, Kim K, Min C-K. The assessment of healthrelated quality of life in relapsed or refractory myeloma patients receiving lenalidomide and low dose dexamethasone: a result of hola study. Blood. 2018;132(Supplement 1):4853.

33. Ludwig H, Pönisch W, Knop S, Egle A, Hinke A, Schreder M, et al. Quality of life in patients with relapsed/refractory multiple myeloma during ixazomibthalidomide-dexamethasone induction and ixazomib maintenance therapy and comparison to the general population. Leuk Lymphoma. 2020;61(2): 377-86. https://doi.org/10.1080/10428194.2019.1666381.

34. Moreau P, Kumar S, Boccia R, lida S, Goldschmidt H, Cocks K, et al. Convenience, satisfaction, health-related quality of life of once-weekly $70 \mathrm{mg} /$ m2 vs. twice-weekly 27 mg/m2 carfilzomib (randomized A.R.R.O.W. study). Leukemia. 2019;33(12):2934-46. https://doi.org/10.1038/s41375-019-0480-2.

35. Moreau P, Masszi T, Grzasko N, Bahlis NJ, Hansson M, Pour L, et al. Oral Ixazomib, lenalidomide, and dexamethasone for multiple myeloma. N Engl J Med. 2016;374(17):1621-34. https://doi.org/10.1056/NEJMoa1516282.

36. Richardson PG, Schlossman RL, Roy AN, Panneerselvam A, Acharyya S, Sopala M, et al. Patient-reported outcomes of multiple myeloma patients treated with panobinostat after $\geq 2$ lines of therapy based on the international phase 3, randomized, double-blind, placebo-controlled PANORAMA-1 trial. Br J Haematol. 2018;181(5):628-36. https://doi.org/1 0.1111/bjh.15248.

37. Stewart AK, Dimopoulos MA, Masszi T, Špička I, Oriol A, Hájek R, et al. Healthrelated quality-of-life results from the open-label, randomized, phase III ASPIRE trial evaluating carfilzomib, lenalidomide, and dexamethasone versus lenalidomide and dexamethasone in patients with relapsed multiple myeloma. JCO. 2016;34(32):3921-30. https://doi.org/10.1200/JCO.2016.66.9648.

38. Weisel K, Paner A, Engelhardt M, Taylor F, Cocks K, Espensen A, et al. Quality-of-life outcomes in patients with relapsed/refractory multiple myeloma treated with elotuzumab plus pomalidomide and dexamethasone: results from the phase 2 randomized eloquent- 3 study. Blood. 2018;132(Supplement 1):2288.

39. Weisel K, Dimopoulos M, Song KW, Moreau P, Palumbo A, Belch A, et al. Pomalidomide and low-dose dexamethasone improves health-related quality of life and prolongs time to worsening in relapsed/refractory patients with multiple myeloma enrolled in the MM-003 randomized phase III trial. Clin Lymphoma Myeloma Leuk. 2015;15(9):519-30. https://doi.org/1 0.1016/j.clml.2015.05.007

40. Askew RL, Xing Y, Palmer JL, Cella D, Moye LA, Cormier JN. Evaluating minimal important differences for the FACT-melanoma quality of life questionnaire. Value Health. 2009;12(8):1144-50. https://doi.org/10.1111/j.1 524-4733.2009.00570.x.

41. Cella D, Nichol MB, Eton D, Nelson JB, Mulani P. Estimating clinically meaningful changes for the functional assessment of cancer therapy - prostate: results from a clinical trial of patients with metastatic hormone-refractory prostate cancer. Value Health. 2009;12(1):124-9. https:// doi.org/10.1111/j.1524-4733.2008.00409.X.

42. Cheung YT, Foo YL, Shwe M, Tan YP, Fan G, Yong WS, et al. Minimal clinically important difference (MCID) for the functional assessment of cancer therapy: cognitive function (FACT-Cog) in breast cancer patients. J Clin Epidemiol. 2014. 67(7):811-20. https://doi.org/10.1016/j.jclinepi.2013.12.011.

43. Yost K, Cella D, Chawla A, Holmgren E, Eton D, Ayanian J, et al. Minimally important differences were estimated for the functional assessment of cancer therapy-colorectal (FACT-C) instrument using a combination of distribution- and anchor-based approaches. J Clin Epidemiol. 2005;58(12): 1241-51. https://doi.org/10.1016/j.jclinepi.2005.07.008.

44. Yost KJ, Sorensen MV, Hahn EA, Glendenning GA, Gnanasakthy A, Cella D. Using multiple anchor- and distribution-based estimates to evaluate clinically meaningful change on the functional assessment of cancer therapy-biologic response modifiers (FACT-BRM) instrument. Value Health. 2005:8(2):117-27. https://doi.org/10.1111/j.1524-4733.2005.08202.x.

45. Dubois D, Dhawan R, van de Velde H, Esseltine D, Gupta S, Viala M, et al. Descriptive and prognostic value of patient-reported outcomes: the Bortezomib experience in relapsed and refractory multiple myeloma. JCO. 2006;24(6):976-82. https://doi.org/10.1200/JCO.2005.04.0824.

46. Wing C, Simon K, Bello-Gomez RA. Designing difference in difference studies: best practices for public health policy research. Annu Rev Public Health. 2018; 39(1):453-69. https://doi.org/10.1146/annurev-publhealth-040617-013507.

\section{Publisher's Note}

Springer Nature remains neutral with regard to jurisdictional claims in published maps and institutional affiliations. 